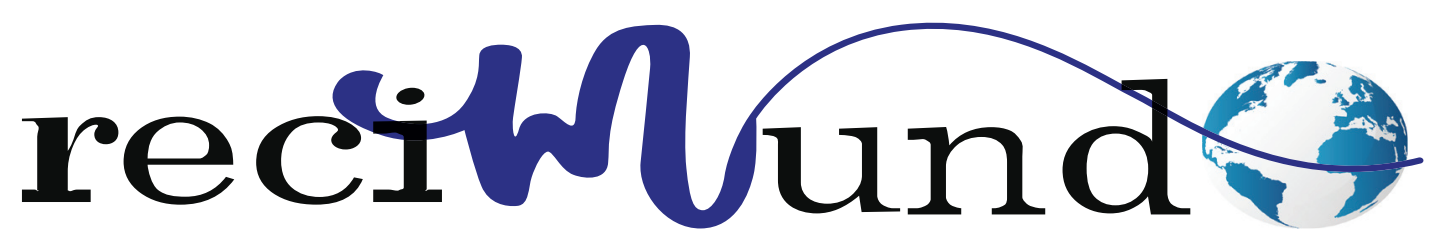

Revista Científica Mundo de la Investigación y el Conocimiento

DOI: $10.26820 /$ recimundo/5.(1).enero.2021.50-56

URL: http://recimundo.com/index.php/es/article/view/982

EDITORIAL: Saberes del Conocimiento

REVISTA: RECIMUNDO

ISSN: 2588-073X

TIPO DE INVESTIGACIÓN: Artículo de Revisión

CÓDIGO UNESCO: 3205 Medicina Interna

PAGINAS: 50-56

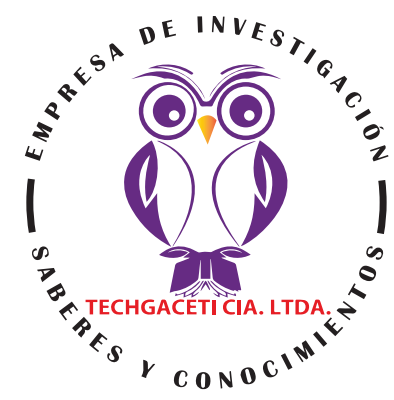

\title{
Manejo de neumotórax espontaneo
}

Management of spontaneous pneumothorax

Gerenciamento do pneumotórax espontâneo

Danilo Francisco Esquivel Ramírez; Elsa Lissette Hernández Negrete; Fanny Katherine Tupiza Luna3;

Christian Andrés Barcia Velásquez ${ }^{4}$

\section{RECIBIDO: 28/11/2020 ACEPTADO: 06/12/2020 PUBLICADO: 31/01/2021}

1. Médico Residente Hospital Baca Ortiz; Quito, Ecuador; danier15@outlook.com; iD https://orcid.org/0000-0002-0416-4606

2. Médico Residente; Investigador Independiente; Quito, Ecuador; ellen1689@hotmail.com; (iD https://orcid.org/0000-00029314-4366

3. Médico Residente Medicina Interna HE-1; Quito, Ecuador; fannykatherine@yahoo.com; (iD https://orcid.org/0000-00024695-9802

4. Médico Residente Cirugía Cardiotorácica HCAM; Quito, Ecuador; chris_barcia@hotmail.com; (iD) https://orcid.org/00000002-8682-0389

\section{CORRESPONDENCIA}

Danilo Francisco Esquivel Ramírez

danier15@outlook.com

Quito, Ecuador

ㄷ RECIMUNDO; Editorial Saberes del Conocimiento, 2021 


\title{
RESUMEN
}

Como lo define la bibliografía, el neumotórax es la acumulación de aire en el espacio pleural, que se puede producir espontáneamente, es decir, por otras afecciones y el no espontaneo que es producido generalmente por un evento traumático. El diagnostico se confirma con la realización generalmente de una radiografía de tórax, sin embargo, estudios más profundas pueden sugerir un TAC torácico o una Gasometría. La revisión médica inicial es determinante para poder identificar qué tipo de neumotórax está presente para de esta manera decidir el tratamiento más adecuado. La metodología de la investigación, es una revisión bibliográfica, apoyada en medios electrónicos como fuente primaria de la obtención de la información. El manejo del neumotórax conlleva dos tipos de tratamientos, el primero que es el terapéutico y el segundo es el quirúrgico que contempla la cirugía convencional o la cirugía videotoracoscópica.

Palabras clave: Cirugía, Terapéutica, Neumotórax, Espontaneo, Pleural.

\begin{abstract}
As the bibliography defines it, pneumothorax is the accumulation of air in the pleural space, which can occur spontaneously, that is, by other conditions and the non-spontaneous one that is generally produced by a traumatic event. The diagnosis is generally confirmed with a chest X-ray, however, deeper studies may suggest a chest CT scan or blood gas analysis. The initial medical check-up is decisive to be able to identify what type of pneumothorax is present in order to decide the most appropriate treatment. The research methodology is a bibliographic review, supported by electronic media as the primary source for obtaining information. The management of pneumothorax involves two types of treatment, the first is therapeutic and the second is surgical, which includes conventional surgery or videothoracoscopic surgery.
\end{abstract}

Keywords: Surgery, Therapeutics, Pneumothorax, Spontaneous, Pleural.

\section{RESUMO}

Como a bibliografia o define, pneumotórax é a acumulação de ar no espaço pleural, que pode ocorrer espontaneamente, ou seja, por outras condições e a não espontânea que geralmente é produzida por um evento traumático. O diagnóstico é geralmente confirmado com uma radiografia de tórax, entretanto, estudos mais profundos podem sugerir uma tomografia de tórax ou uma análise de gases sanguíneos. O exame médico inicial é decisivo para poder identificar que tipo de pneumotórax está presente a fim de decidir o tratamento mais apropriado. A metodologia de pesquisa é uma revisão bibliográfica, apoiada por meios eletrônicos como a principal fonte para a obtenção de informações. O gerenciamento do pneumotórax envolve dois tipos de tratamento, o primeiro é terapêutico e o segundo é cirúrgico, que inclui cirurgia convencional ou cirurgia videotoracoscópica.

Palavras-chave: Cirurgia, Terapêutica, Pneumotórax, Espontânea, Pleural. 


\section{Introducción}

La acumulación de aire en el espacio pleural entraña una situación patológica que se denomina neumotórax. Puede darse sin que medie traumatismo, lo que se conoce como "neumotórax espontáneo", donde subyace una patología a nivel del espacio aéreo: burbujas o blefs (se ven en menores de 20 años) o rotura de bullas enfisematosas (epoc, ancianos), que generalmente son de localización apical y periféricos, donde la rotura de la pleura visceral, hace que el aire del espacio aéreo gane el espacio pleural y se acumule originando dicha patología. En la mayoría de los casos media un evento traumático, ya sea por herida punzante o de arma de fuego, por fracturas costales por impacto directo o bien de causa iatrogénica durante métodos diagnósticos o terapéuticos de punción pulmonar para biopsia o avenamiento o colocación de catéteres de vías centrales, en especial subclavia (Featherson, y otros, 2012).

La clasificación actual divide al neumotórax en espontáneo o no espontáneo (traumático), subdividiendo la primera categoría en espontáneo primario, espontáneo secundario o catamenial (asociado a la menstruación), siendo el espontáneo primario definido como aquel que ocurre en pacientes sin un evento traumático precipitante y/o sin condiciones pulmonares patológicas previas (González-Hermosillo-Cornejo, Díaz-Girón-Gidi, \& Vidal-Tamayo, 2015).

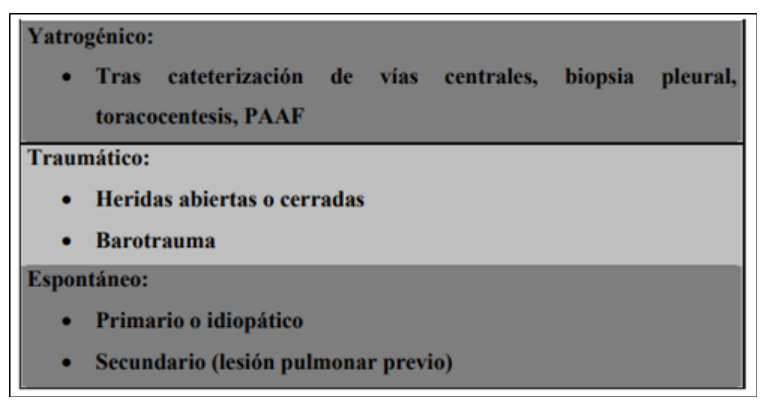

Figura 1. Clasificación del neumotórax

Fuente: (Jiménez, en Neumología, \& Rios, 2000).
El neumotórax traumático se divide en cerrado o abierto en función de la presencia o no de herida penetrante, con mucha frecuencia se acompaña de hemotórax de cuantía variable. El barotrauma suele darse en pacientes sometidos a ventilación mecánica, relacionándose este hecho con el uso de volúmenes corrientes y PEEP elevados. Si no nos encontramos ante una causa clara estamos ante un neumotórax espontáneo (Jiménez, en Neumología, \& Rios, 2000).

A/ Hablamos de neumotórax espontáneo primario si no encontramos ninguna causa pulmonar de base que lo justifique.

B/ neumotórax espontáneo secundario, encontramos diversas situaciones patológicas con afectación pulmonar, como son:

a) Asma: suele coincidir con las agudizaciones y con frecuencia se acompaña de neumomediastino.

b) EPOC: con frecuencia son secundarios a la rotura de bullas intrapulmonares. Suele cursar con importante afectación general y es conveniente sospecharla en todo EPOC con aumento brusco de su disnea crónica y dolor pleurítico.

c) Fibrosis pulmonares avanzadas: en aquellas que existe panalizacion y bullas como en el granuloma eosinófilo.

d) Enfermedades del tejido conectivo como síndrome de Marfan y EhlersDanlos.

e) Neumotórax catamenial es una entidad rara que se da en mujeres de mediana edad en el contexto de la menstruación y su sustrato anatomopatológicos corresponde a un foco endometriósico ectópico.

f) En cuanto a las causas infecciosas hay que destacar las neumonías necrotizantes en especial la originada por el estafilococo, la tuberculosis que puede originar focos caseosos subpleurales, el Pneumocystis Carinii.

g) Otras asociaciones son lo la fibrosis 
quísticas, neumonías aspirativas, infecciones fúngicas, sarcoidosis, carcinoma broncogenico, metástasis, etc. (Jiménez, en Neumología, \& Rios, 2000).

\section{Etiología}

En el caso del Neumotórax Espontáneo de tipo primario, al no contar con una enfermedad desencadenante, esta entidad se relaciona con factores de riesgo predisponentes como los son el fumado, antecedentes familiares, síndrome de Marfán, homocicteinuria, anorexia y endometriosis intratorácica. El fumado es el factor de riesgo principal, ya que estudios en paciente con neumotórax espontáneo primario, hasta en un $90 \%$ eran fumadores (Valle Sagastume, 2016).

En los antecedentes familiares se han propuesto múltiples alteraciones genéticas, la más conocida es el Síndrome de Birt-Hogg-Dubé, que consiste en una mutación de tipo autosómico dominante que predispone a fibrofoliculomas (tumores benignos de la piel), quistes pulmonares y cáncer renal (Valle Sagastume, 2016).

El Neumotórax Espontáneo secundario, es ocasionado por a la enfermedad pulmonar subyacente como lo es la enfermedad pulmonar obstructiva crónica (EPOC), Fibrosis Quística, malignidad primaria o metastásica y Neumonía Necrotizante. El EPOC es la causa más común, siéndolo hasta en un 50$70 \%$, el mecanismo se produce por ruptura de una bulla apical del pulmón. Los paciente con Fibrosis Quística, en un 5\% aproximadamente, presentarán un neumotórax espontáneo (Valle Sagastume, 2016).

Pacientes con Cáncer pulmonar primario o metastásico representan un riesgo elevado de presentar neumotórax, ya que se produce necrosis tumoral y obstrucción endobronquial que produce atrapamiento de aire (Valle Sagastume, 2016).

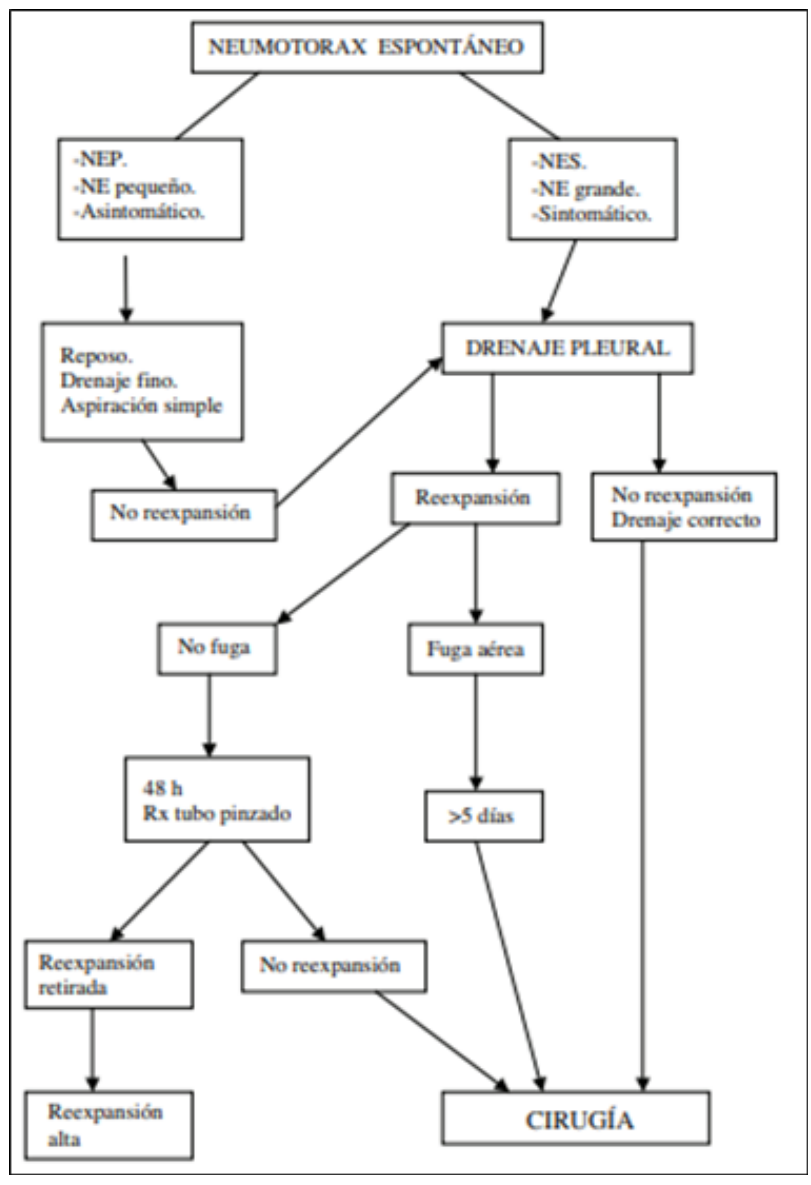

Figura 2. Algoritmo terapéutico del neumotórax espontáneo

Fuente: (García \& Algar, 2007)

\section{Clínica del neumotórax}

La sintomatología es variada, aunque lo más típico suele ser el dolor torácico agudo de características pleuríticas. La disnea, que varía en función del grado de afectación pulmonar previa. También pueden aparecer tos seca y hemoptisis. Incluso hay pacientes asintomáticos en los que el neumotórax puede ser un hallazgo casual. La severidad de la sintomatología (dolor, disnea, tos seca) va correlacionada con la reserva funcional del pulmón contralateral y con el grado de colapso pulmonar (García \& Algar, 2007).

\section{Diagnostico}

- Radiografía de tórax: Habitualmente con una radiografía P-A de tórax en inspira-

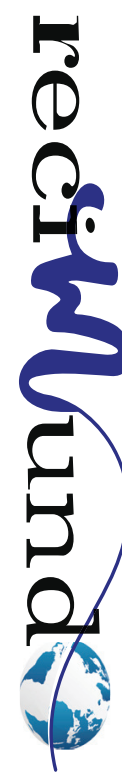


ción podremos comprobar la existencia o no de neumotórax. Sólo cuando existan dudas en neumotórax pequeños se solicitará una radiografía P-A de tórax en espiración. La evidencia de una línea pleural visceral separada de la parietal, sin trama vascular, únicamente por un espacio hiperclaro confirma el diagnóstico de neumotórax.

- TAC torácico: Es muy útil para diferenciar el neumotórax de la bulla de enfisema, además informa de la cuantía real del tamaño del neumotórax. La TAC torácica se debe reservar para los casos de duda de la existencia de neumotórax.

- Gasometría: Puede aparecer hipoxemia y en ocasiones hipocapnia si el paciente está taquipneico (García \& Algar, 2007).

Existen cuadros que presentan una sintomatología similar al neumotórax (dolor torácico y disnea) como son: derrame pleural, tromboembolismo pulmonar, disección aórtica, úlcera péptica perforada, cardiopatía isquémica, pericarditis y rotura esofágica. Mediante una simple radiografía de tórax podemos confirmar el diagnóstico de neumotórax. Únicamente en la rotura esofágica se puede evidenciar un neumotórax en la radiografía (García \& Algar, 2007).

\section{Metodología}

La metodología de la investigación, es una revisión bibliográfica, apoyada en medios electrónicos como libros, artículos, páginas alojadas en la web, como fuentes primarias de la recolección y análisis de la información. Lo que permitió tener una perspectiva más amplia y completa del tema en estudio, gracias a la bibliográfica consultada.

\section{Resultados}

\section{Manejo del neumotórax}

Todavía no existe una unificación de criterios sobre que tratamiento es el más idóneo, por lo que trataremos de sistematizar los tratamientos más aceptados actualmente (Doménech, 2006).

En el primer episodio de neumotórax:

- Reposo: La cavidad pleural tiene una capacidad de absorción de aire; deberíamos sólo aplicar este tratamiento en aquellos neumotórax espontáneos pequeños, con mínima o nula repercusión clínica, controlándose en régimen ambulatorio.

- Drenaje torácico: Consiste en la introducción de un drenaje en el espacio pleural del hemitórax afecto de forma aséptica: podremos utilizar drenajes de pequeño diámetro (catéteres de $8 \mathrm{~F}$ ) o tubos de mayor diámetro (20-28F). El lugar de colocación siempre irá encaminado a evacuar totalmente el neumotórax y así obtener la reexpansión total pulmonar: de forma estandarizada podemos decir que se introducirían en el segundo espacio intercostal, en la línea media clavicular. También en quinto espacio intercostal, en la línea anterior o línea media axilar siempre que exista cámara de neumotórax en estas zonas. En algunos casos con procesos adherenciales tendríamos que introducirlo en la zona donde se encuentre la cámara aérea observando el no lesionar el parénquima pulmonar subyacente.

- Cirugía: Actualmente se propone como tratamiento de elección del neumotórax espontáneo en una serie de situaciones clínicas que se mencionan a continuación: neumotórax espontáneo recidivante, neumotórax espontáneo primer episodio que ha sido tratado mediante drenaje y mantiene fugas aéreas durante un espacio de tiempo superior a 7 días, hemoneumotórax importante, neumotórax espontáneo que tiene recidiva intrahospitalaria, neumotórax en pacientes que por su profesión pueden tener problemas para ser atendidos en un hospital (pilotos de líneas aéreas, deportistas, conductores de camiones, 
marinos), neumotórax espontáneo con lesiones que dificulten su reexpansión, neumotórax espontáneo bilateral simultaneo, neumotórax espontáneo primer episodio contralateral y neumotórax espontáneo a tensión. También se puede indicar la cirugía en aquellos casos de neumotórax espontáneo con patología radiológica visible (blebs o bullas) (Doménech, 2006).

La cirugía videotoracoscópica será la primera opción para el tratamiento quirúrgico del neumotórax espontáneo: Se lleva a cabo a través de tres puertos o puertas de entrada por donde se introduce la lente que lleva acoplada una videocámara que trasmite la imagen a un monitor de TV y por los otros puertos se introducirá el instrumental apropiado y diseñado para poder extirpar la patología pleuropulmonar causante del neumotórax espontáneo. Posteriormente se suele añadir algún tipo de maniobra que permita crear un cuadro adherencial pleuropulmonar. Entre éstos podemos mencionar: abrasión de pleura parietal con gasas secas, abrasión de pleura parietal con esponjillas metálicas, escarificación de pleura parietal con electro bisturí láser, pleurectomía parietal parcial apical, pleurodesis con cola de fibrina, pleurodesis química con talco. Esta última se debe reservar solo para pacientes adultos con neumotórax espontáneo secundario, aunque hay grupos que lo utilizan también en pacientes con neumotórax espontáneo primario (Doménech, 2006). La cirugía convencional se lleva a cabo a través de toracotomías de pequeña longitud anteriores (axilares), laterales o posteriores. Como vía de acceso alternativa para poder tratar lesiones bilaterales en un mismo acto quirúrgico y cuando no se puede realizar con cirugía videotoracoscópica, podremos realizar toracotomías transesternales, toracotomía bilateral secuencial y toracotomía bilateral transesternal. Este último tipo de abordaje presenta una alta tasa de morbilidad (Doménech, 2006).
Pleurodesis: En aquellos casos de neumotórax espontáneo que la situación del paciente no permita poder someterlo a un tratamiento definitivo mediante cirugía, existe la alternativa de conseguir que se produzca un cuadro adherencial pleuropulmonar permanente y así evitar en lo posible la recidiva. La condición indispensable e ideal es que el pulmón esté expandido totalmente y que la dos pleuras estén en contacto para obtener un buen resultado: como agentes sinfisiantes se han utilizado sustancias químicas como: clorhidrato de tetraciclina, bleomicina, y polvo inorgánico (talco) (Doménech, 2006).

\section{Conclusiones}

El neumotórax como se ha podido leer en la presente investigación, es un evento que se puede producir por varios factores, de allí que lo clasifican en primario y secundario, lo importante de ello es saber identificar a tiempo que tipo de neumotórax padece el paciente, si efectivamente es un neumotórax, para iniciar el tratamiento adecuado.

El manejo del neumotórax va a depender de la gravedad que padezca el paciente, se tiene como técnica la terapéutica y la quirúrgica. La terapéutica va desde el reposo hasta el drenaje torácico y la quirúrgica comprende la cirugía convencional y la cirugía videotoracoscópica.

La detección del neumotórax a simple vista, el medico la puede ir detectando mediante la exploración del paciente, luego la comprobación se realiza mediante una radiografía de tórax. Estudios más profundos requieren TAC torácico y gasometría.

\section{Bibliografía}

Doménech, A. B. (2006). Manejo del neumotórax. Revista Neumosur, 18(4), 198-201.

Featherson, C., Paglia, A., Riquelme, R., Tomasco, F., Camila, R., Santin, D., \& Michelini, C. (2012). Neumotórax, manejo clínico quirúrgico. Tercera Época, 3(2), 1-2. 
García, J. C., \& Algar, F. (2007). Diagnóstico y manejo terapéutico del neumotórax. Revista Neumosur, 19(3), 138-142.

González-Hermosillo-Cornejo, D., Díaz-Girón-Gidi, A., \& Vidal-Tamayo, R. (2015). Neumotórax espontáneo primario, revisión de la literatura. Médica Sur, 22(1), 19-26.
Jiménez, M. D., en Neumología, C., \& Rios, C. (2000). Manejo Del Neumotorax. Medynet. Com, 1-31.

Valle Sagastume, J. C. (2016). Neumotórax espontáneo. Revista Médica de Costa Rica y Centroamérica, 72(617), 723-727.

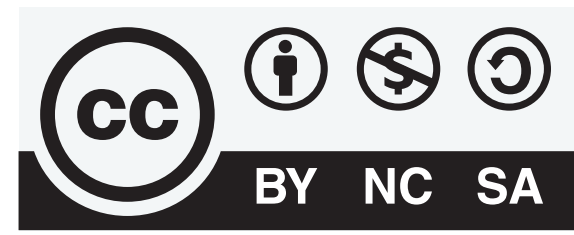

CREATIVE COMMONS RECONOCIMIENTO-NOCOMERCIAL-COMPARTIRIGUAL 4.0.

\section{CITAR ESTE ARTICULO:}

Esquivel Ramírez, D. F., Hernández Negrete, E. L., Tupiza Luna, F. K., \& Barcia Velásquez, C. A. (2021). Manejo de neumotórax espontaneo. RECIMUNDO, 5(1), 50-56. https://doi.org/10.26820/recimundo/5.(1).enero.2021.50-56 